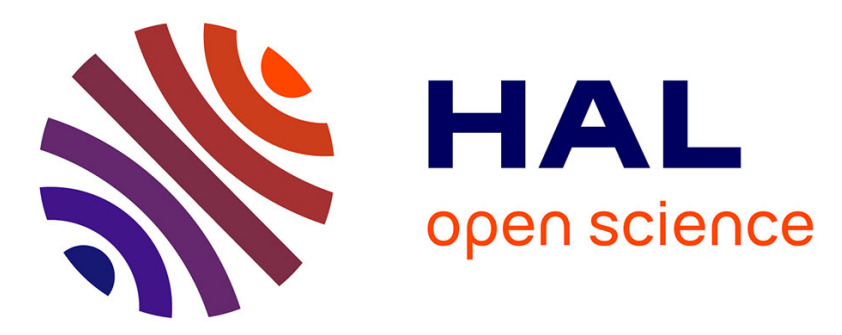

\title{
Network sharing by two mobile operators: beyond competition, cooperation
}

\author{
Alexandre Blogowski, Philippe Chrétienne, Fanny Pascual
}

\section{To cite this version:}

Alexandre Blogowski, Philippe Chrétienne, Fanny Pascual. Network sharing by two mobile operators: beyond competition, cooperation. RAIRO - Operations Research, 2015, 10.1051/ro/2014061 . hal01317570

\section{HAL Id: hal-01317570 https://hal.sorbonne-universite.fr/hal-01317570}

Submitted on 15 Dec 2021

HAL is a multi-disciplinary open access archive for the deposit and dissemination of scientific research documents, whether they are published or not. The documents may come from teaching and research institutions in France or abroad, or from public or private research centers.
L'archive ouverte pluridisciplinaire HAL, est destinée au dépôt et à la diffusion de documents scientifiques de niveau recherche, publiés ou non, émanant des établissements d'enseignement et de recherche français ou étrangers, des laboratoires publics ou privés. 


\title{
RAIRO Operations Research
}

Will be set by the publisher

\section{NETWORK SHARING BY TWO MOBILE OPERATORS: BEYOND COMPETITION, COOPERATION}

\author{
Alexandre Blogowski $^{a, b}$, Philippe Chrétienne ${ }^{a}$, FAnny \\ PASCUAL $^{a 1}$
}

\begin{abstract}
In this paper, we study the sharing of a radio access network infrastructure by two mobile operators. Knowing the possible locations of the base stations, each operator chooses to invest or not on a base station, and its aim is to maximize its profit. We characterize the existence of Nash equilibria in such a game and we measure their quality with respect to the maximization of the overall profit (with the price of anarchy/stability). We then show how to obtain a solution in which each operator earns at least as much as it would have earned in any Nash equilibrium. Finally we conduct experiments on randomly generated instances and on real data.
\end{abstract}

\section{INTRODUCTION}

The mobile phone plays a crucial role in making many services available to the population. In developing countries, there remains much to be done to cover rural areas. The whole problem stems from the high cost of network infrastructure, resulting in high prices charged by the operators, who seek to recoup their investments. Infrastructure sharing for mobile services is a solution that allows to reduce this cost. Many countries already share mobile infrastructure. For example, Orange and Vodafone have an agreement in the UK and Spain, and there are cooperation in countries such as Brazil, Jordan, Canada, India and Malaysia.

There are two broad categories of infrastructure sharing in mobile services: active sharing and passive sharing. The active sharing implies sharing elements of the

\footnotetext{
$02 / 19 / 2014$.
}

1 (a) LIP6, Université Pierre et Marie Curie, 4 place Jussieu, 75005 Paris, France; (b) Orange Labs, France Télécom, 38 rue du Général Leclerc, 92130 Issy-les-Moulineaux, France; alexandre.blogowski@orange.com, \{philippe.chretienne, fanny.pascual\}@lip6.fr 
active layer of mobile networks, such as elements of the core network, and also includes mobile roaming, which allows an operator to use the network of another operator where it does not have an antenna. The passive sharing is related to the sharing of physical spaces, such as buildings, sites and towers, while the networks remain separate. We focus in this paper on this second case.

We study the network sharing of an infrastructure known by two operators in competition. Knowing the existing network infrastructure, namely the possible locations of the base stations (also called sites), the aim of each operator is to determine its optimal deployment strategy in order to maximize its profit. Its profit is usually equal to the revenue gained by the operator thanks to the subscriptions of clients covered by the sites where it invests, minus the costs of investment. The profit earned on a given site may be different for each operator, and the profit of a given operator on a given site may also be different if it is the only one to invest on the site or if the other operator also invests on the same site (when both operators co-invest, the number of clients may decrease for each operator, but the cost of investment will also probably decrease). We consider in this paper a general setting where the profits of the operators on each site, when an operator invests alone or when it co-invests, are arbitrary values.

We consider the game in which two operators invest - or not - on a set of sites. Our aim is twofold. From one part, our aim is to analyze the loss of profit due to the competition between the two operators. From another part, our aim is to propose to the operators a solution in which each operator earns as much as it would earn in a competition setting (in a Nash equilibrium). This solution should be as good as possible with respect to the global profit.

Model. The game that we study is the following one. We consider two mobile operators $A$ and $B$ (also called agents), and $n$ sites (base stations). Agent $j \in\{A, B\}$ has for each site $i$ two possible strategies:

- either it invests, and

- if the other agent does not invest on site $i$, then the profit of Agent $j$ is a fixed profit $s_{i}^{j}$ (where $s$ stands for "single").

- if the other agent also invests on site $i$, then the profit of Agent $j$ is a fixed profit $t_{i}^{j}$ (where $t$ stands for "together").

- or it does not invest, and then it has a profit equal to 0 .

We note $x_{i}^{j}=1$ if Agent $j$ invests on site $i, 0$ otherwise. We have $\overline{x_{i}^{j}}=1-x_{i}^{j}$. The profit of Agent $A$ (resp. $B$ ), denoted by $\operatorname{Prof}^{A}$ (resp. Prof ${ }^{B}$ ), is the sum of the profits it gains at each site:

$$
\operatorname{Prof}^{A}=\sum_{i=1}^{n}\left(x_{i}^{A} \overline{x_{i}^{B}} s_{i}^{A}+x_{i}^{A} x_{i}^{B} t_{i}^{A}\right) \quad\left(\operatorname{resp} . \operatorname{Prof}^{B}=\sum_{i=1}^{n}\left(\overline{x_{i}^{A}} x_{i}^{B} s_{i}^{B}+x_{i}^{A} x_{i}^{B} t_{i}^{B}\right)\right)
$$

The aim of each agent is to maximize its profit. The social cost that we consider is the maximization of the overall profit (i.e. $\operatorname{Prof}^{A}+\operatorname{Prof}^{B}$ ). We denote this 
objective function by MaxSP (where SP stands for "sum of profits"). As each site $i$ is independent, maximizing the overall profit may be obtained by taking the maximum locally on each site $i$ and then summing up these values. Thus, the social cost function is:

$$
\operatorname{maximize} \sum_{i=1}^{n}\left(x_{i}^{A} \overline{x_{i}^{B}} s_{i}^{A}+\overline{x_{i}^{A}} x_{i}^{B} s_{i}^{B}+x_{i}^{A} x_{i}^{B}\left(t_{i}^{A}+t_{i}^{B}\right)\right)
$$

In a realistic context, an additional constraint of population coverage must be taken into account. This constraint is imposed by the regulations of the country: each agent $j$ must cover at least a given fraction $C^{j}$ of the population ${ }^{1}$. We denote by $n_{i}$ the number of persons (subscribers or not) present in the scope of the site $i$. Each agent which invests on the site $i$ will cover these $n_{i}$ persons. We denote by $\operatorname{MaxSP}(\mathrm{cov})$ the problem MaxSP in which each Agent $j$ has to cover at least a given fraction $C^{j}$ of the population (i.e. $\frac{\sum_{i=1}^{n} x_{i}^{j} n_{i}}{\sum_{i=1}^{n} n_{i}}$ has to be larger than or equal to $\left.C^{j}\right)$.

In an environment where each agent knows its own profit according to the decisions of all agents, a (pure) Nash equilibrium is a combination of (deterministic) choices, one for each agent, in which no agent can improve its own profit by unilaterally changing its strategy $[9]^{2}$. In our game, a solution is thus a Nash equilibrium if each agent cannot increase its profit by investing on one or several sites in which it does not invest, or by stopping to invest on one or several sites in which it invests. In order to measure the quality of Nash equilibria, we will study the price of anarchy [8] and the price of stability [1,15]. The price of anarchy (resp. price of stability) is the maximum ratio, over all the instances, between the value of the social cost in an optimal solution and the value of the social cost in the worst (resp. best) Nash equilibrium. The price of anarchy is interpreted as the loss of profit due to the lack of cooperation, whereas the price of stability can be interpreted as the lost of profit due to the fact that the agents optimize their own objective functions rather than the social cost. It is expected that the loss in efficiency due to game-theoretical constraints is somewhere between the price of anarchy and the price of stability.

We will study the price of stability and the price of anarchy with respect to the social cost functions MaxSP and $\operatorname{MaxSP}(\mathrm{cov})$, in the case where the profits are either strictly positive or arbitrary, and in these three different cases:

- General case: the profits $s_{i}^{A}, s_{i}^{B}, t_{i}^{A}$ and $t_{i}^{B}$ are arbitrary values in $\mathbb{Z}$.

\footnotetext{
${ }^{1}$ In France, the regulatory authority for electronic communications and postal services (ARCEP in french) verifies operators' compliance with coverage obligations. In July 2012, ARCEP carried out checks on the 3G coverage achieved by France's four 3G mobile operators: Free Mobile, Orange France, SFR and Bouygues Telecom. http://www.arcep.fr/index.php?id=8161

${ }^{2}$ We are interested in this paper only by pure Nash equilibria. In the sequel, we will omit the adjective pure.
} 
- Case $R$ (where $\mathrm{R}$ stands for "realistic"): for any site $i \in\{1, \ldots, n\}$, it is not possible to have the configuration $\left(s_{i}^{A}>0, s_{i}^{B}>0, t_{i}^{A} \leq 0\right.$ and $\left.t_{i}^{B} \leq 0\right)$. In this case, it is not possible that both operators have a positive profit when they invest alone on a site $i$ whereas, when both operators co-invest on this same site, they both have a negative (or zero) profit.

- Case IC (where IC stands for "interest to co-invest"): for any site $i \in\{1, \ldots, n\},\left(t_{i}^{A}+t_{i}^{B}\right) \geq \max \left(s_{i}^{A}, s_{i}^{B}\right)$. In this case, the total profit obtained when both operators co-invest is larger than or equal to the maximum profit of an operator alone on the same site (this case happens when the cost of investment decreases when both operators co-invest while the number of clients and their subscriptions do not decrease).

Moreover, we define the best Nash equilibrium of an agent $j \in\{A, B\}$ as the Nash equilibrium in which the profit of $j$ is maximized.

Our contribution. In Section 1 we give complexity results concerning the problems MaxSP and MaxSP(cov), and we study the existence and the quality of Nash equilibria for these problems. In Section 2, we introduce the problem $\operatorname{MaxSP}($ pmin), where the global profit has to be maximized under the constraint that each agent earns at least as much as it would have received in its best Nash equilibrium. We show that there is always such a solution in the case R; we show that this problem is NP-hard, and we use dynamic programming to solve it. In Section 3, we show simulations results obtained for real data and for randomly generated instances in order to analyze in practice the price of stability/anarchy and the quality of the solutions returned by $\operatorname{MaxSP}(\mathrm{pmin})$. We conclude the paper in Section 4.

Related work. Numerous technical analysis of the gain obtained when several operators share their resources have been done. Bartlett and Jackson [2] measure the total infrastructure and operational cost savings due to a sharing scenario (site sharing, diverse equipment sharing, core network sharing) by comparison to a "non shared" reference network. The indicative savings are based on the assumption that the operators share the costs equally. They conclude that the case of site sharing is the best. More recently, the consulting firm Analysys Mason [10] studies the cost savings when two mobile telecommunication operators build a new network together, and they show that the operators can save up almost half of their development and maintenance costs.

Most papers concerning network sharing between selfish operators use cooperative game theory [12], and consider that the utility of the agents is transferable. Saad et al. [14] provide a comprehensive overview of coalitional game theory and its usage in wireless and communications networks. Contrarily to these works, in this paper we will consider that the utility of the agents is not transferable, since the regulation of the countries often prohibit money transfers between the operators (allowing money transfers would allow the operators to behave such as they were a single operator in situation of monopoly). 
Our work is related to other works which consider selfish organizations, each organization owning resources and clients willing to use these resources. Each organization has its own objective function that it wishes to optimizes. The aim is to design an algorithm which assigns the resources of all the organizations to all the clients, in such a way that each organization has a profit (with respect to its own objective function) as least as good as the profit it would have obtained if it had assigned its own clients to its own resources. This algorithm should maximize a social cost function. Note that in this model the utilities are not transferable. This setting has been introduced by Pascual et al. [13] for a scheduling problem where the resources are the machines and the clients are the tasks (the aim of each organization is to minimize the last completion time of its tasks), and has been followed by several other papers $[3-5,11]$. The authors give approximation algorithms and experimental results for this problem. Gourvès et al. [7] study the complexity and the approximation of a matching problem in which each organization owns vertices of a weighted bipartite graph (resources, clients), and the profit of one organization is a fraction of each edge which has an endpoint in the organization in the returned matching (the social cost is the weight of the matching). We also consider a bipartite graph, but here the agents do not own the resources (the sites), and we will look for a solution in which each agent earns at least what it would have had in any Nash equilibrium, which is another way to make the agents accept the solution.

\section{Problem MaxSP without Cooperation}

The complexity of problems MaxSP and MaxSP(cov) is presented in Subsection 1.1. Subsection 1.2 is devoted to the existence and the quality of Nash equilibria.

\subsection{Complexity}

Problem MaxSP is easy. Indeed, since the sites are independent, the maximum social profit is the sum $\sum_{i=1}^{n} \max \left\{s_{i}^{A}, s_{i}^{B}, t_{i}^{A}+t_{i}^{B}, 0\right\}$ of the $n$ local maximum profits. However, when the coverage constraint is considered, the corresponding problem MaxSP(cov) is NP-hard:

Proposition 1. The decision version of problem MaxSP(cov) is NP-complete, even if all the profits are non negative.

Proof. We show that the PARTITION problem polynomially reduces to our problem. We recall the PARTITION problem [6]:

Let $E$ be a finite set of $n$ elements, and $c(e) \in \mathbb{N}$ be the size of element $e \in E$. It is assumed that $\sum_{e \in E} c(e)=2 K$. The question is: is there a subset $E^{\prime} \subseteq E$ such that $\sum_{e \in E^{\prime}} c(e)=\sum_{e \in E \backslash E^{\prime}} c(e)$ ?

Let $(E, c)$ be an instance of the PARTITION problem. The corresponding instance of $\operatorname{MaxSP}(\mathrm{cov})$ is as follows: with each element $e \in E$ is associated a site $\widehat{e}$ with a population $n_{\widehat{e}}=c(\widehat{e})$. Moreover, we define for any site $\widehat{e}$ the following profits 
$s_{\widehat{e}}^{A}=s_{\widehat{e}}^{B}=1$ and $t_{\widehat{e}}^{A}=t_{\widehat{e}}^{B}=0$. We assume that each agent must cover at least $K$ clients and that the total profit must be at least $n$.

If $(E, c)$ is a yes instance such that $\sum_{e \in E^{\prime}} c(e)=K$, then there is a solution of the corresponding instance of the problem MaxSP(cov) where Agent $A$ invests on the sites of $E^{\prime}$ and not on the other sites, and Agent $B$ invests on the sites of $E \backslash E^{\prime}$ and not on the other sites. Each agent covers exactly $K$ clients and the profit of the solution is $n$. Conversely, if the cost of the solution of the instance of the problem MaxSP(cov) is $n$, then this means that each site is covered by exactly one agent, and thus that each agent covers exactly $K$ clients: so there is a solution to the $(E, c)$ instance of the PARTition problem.

Note that, in the case IC and if all the profits are non negative, problem MaxSP(cov) is easy: the optimal solution is indeed obtained when both operators co-invest on all the sites.

\subsection{Existence AND QUALity of Nash EQUiLibria}

For problems MaxSP and MaxSP(cov), when all the profits are strictly positive, each agent has incentive to invest on each site. The solution where both agents co-invest on each site is thus a Nash equilibrium. If some profits are negative, there may be cases where there is no Nash equilibrium. Proposition 2 characterizes the instances where a Nash equilibrium exists when the agents do not have to cover a given fraction of the population.

Proposition 2. If the agents do not have a coverage constraint, for a given instance, there is no Nash equilibrium if and only if there exists a site $i$ such that

$$
\left(s_{i}^{A}<0<t_{i}^{A} \text { and } t_{i}^{B}<0<s_{i}^{B}\right) \text { or }\left(t_{i}^{A}<0<s_{i}^{A} \text { and } s_{i}^{B}<0<t_{i}^{B}\right)
$$

Proof. Since the sites are independent, there is no Nash equilibrium if and only if there exists a site $i$ such that there is no Nash equilibrium for the instance where all the sites except $i$ are removed. We will thus focus on a site $i$ and show that there is no Nash equilibrium for the site $i$ alone if and only if $\left(s_{i}^{A}<0<t_{i}^{A}\right.$ and $\left.t_{i}^{B}<0<s_{i}^{B}\right)$ or $\left(t_{i}^{A}<0<s_{i}^{A}\right.$ and $\left.s_{i}^{B}<0<t_{i}^{B}\right)$.

Assume first that $\left(s_{i}^{A}<0<t_{i}^{A}\right.$ and $\left.t_{i}^{B}<0<s_{i}^{B}\right)$ or $\left(t_{i}^{A}<0<s_{i}^{A}\right.$ and $\left.s_{i}^{B}<0<t_{i}^{B}\right)$. As we can see on Figure 1.2, we have four possible cases. With the configuration of the profits above, for each of these cases, at least one agent can improve its profit by changing its strategy (the arrows in Figure 1.2 indicate a cycle when $\left(t_{i}^{A}<0<s_{i}^{A}\right.$ and $\left.s_{i}^{B}<0<t_{i}^{B}\right)$; when $\left(s_{i}^{A}<0<t_{i}^{A}\right.$ and $\left.t_{i}^{B}<0<s_{i}^{B}\right)$, the cycle is in the other direction - clockwise). Thus, there is no Nash equilibrium.

Assume now that there is no Nash equilibrium. The case (Agent $A$ invests, Agent $B$ invests) in Figure 1.2 is thus not a Nash equilibrium. This implies that at least one agent can improve its profit $\left(t_{i}^{A}\right.$ or $\left.t_{i}^{B}\right)$ by changing its strategy. So either Agent $A$ or Agent $B$ should stop investing. We show that the first case implies 
$\left(t_{i}^{A}<0<s_{i}^{A}\right.$ and $\left.s_{i}^{B}<0<t_{i}^{B}\right)$, and that the second case implies $\left(s_{i}^{A}<0<t_{i}^{A}\right.$ and $t_{i}^{B}<0<s_{i}^{B}$ ). Consider the first case (indicated by the arrows on Figure 1.2). If Agent $A$ can improve its profit by changing its strategy, then Agent $A$ does not invest and perceives a null profit. This implies that $t_{i}^{A}$ is a negative profit. Since there is no Nash equilibrium, the configuration (Agent $A$ does not invest, Agent $B$ invests) is also not a Nash equilibrium. Agent $A$ cannot improve its profit because $t_{i}^{A}<0$, so Agent $B$ must improve its profit by not investing on the site $i$. If it does not invest on the site, Agent $B$ perceives a null profit, and thus $s_{i}^{B}$ is a negative profit.

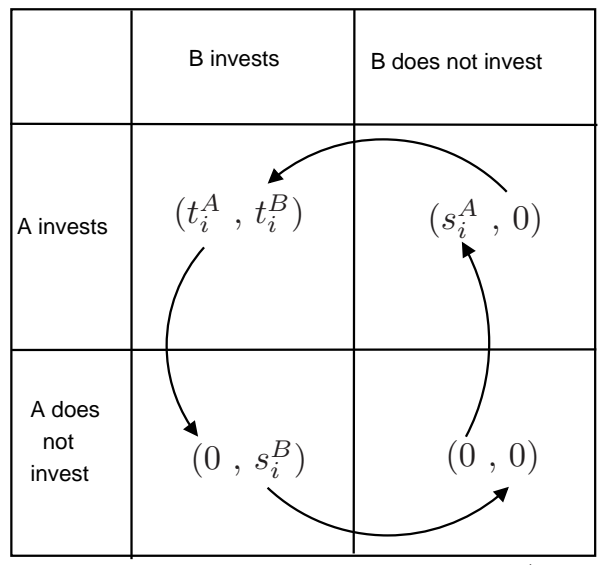

Figure 1. The left hand side (resp. right hand side) of the couple is the profit of Agent $A$ (resp. $B$ ). The arrows indicate a cycle when $\left(t_{i}^{A}<0<s_{i}^{A}\right.$ and $\left.s_{i}^{B}<0<t_{i}^{B}\right)$
Likewise, the configuration (Agent $A$ does not invest, Agent $B$ does not invest) is not a Nash equilibrium: thus Agent $A$ improves its profit by investing on the site $i$. So $s_{i}^{A}$ is a positive profit. Similarly, the configuration (Agent $A$ invests, Agent $B$ does not invest) is not a Nash equilibrium: Agent $B$ improves its profit by investing on the site $i$. So $t_{i}^{B}$ is a positive profit. Therefore, in this first case the configuration of the profits of both agents is as follows: $\left(t_{i}^{A}<0<s_{i}^{A}\right.$ and $\left.s_{i}^{B}<0<t_{i}^{B}\right)$.

For the second case, where Agent $B$ has incentive to stop investing, starting from the case (Agent $A$ invests, Agent $B$ invests), the proof is similar. This case leads to the following configuration of profits: $\left(s_{i}^{A}<0<t_{i}^{A}\right.$ and $\left.t_{i}^{B}<0<s_{i}^{B}\right)$.

In practice, to our knowledge and after studying some real data (see Section 3), all the instances have a Nash equilibrium. So, in the sequel, we will consider instances that do have a Nash equilibrium. We now study the quality of these Nash equilibria.

Proposition 3. The price of stability for problems MaxSP and MaxSP(cov) is unbounded, even in the case IC. Likewise the price of stability is unbounded, even when all the profits are strictly positive.

Proof. Let $M$ be an arbitrary large integer. We consider an instance with only one site 1 , and where the profits are $s_{1}^{A}=-1, t_{1}^{A}=-1, s_{1}^{B}=1$ and $t_{1}^{B}=M$. These profits verify the condition IC, and the only Nash equilibrium is when Agent $B$ is the only one that invests. The sum of profits is then 1 . However, the optimal sum 
of the profits is $M$ when both agents co-invest. The price of stability is then $M$.

Let us now consider the following instance where there is one site, and where the (strictly positive) profits are: $s_{1}^{A}=M, t_{1}^{A}=1, s_{1}^{B}=M$ and $t_{1}^{B}=1$. The only Nash equilibrium is when Agents $A$ and $B$ co-invest. The sum of the profits is 2 , while the optimal sum of the profits is $M$ when only one operator invests. The price of stability is then $M / 2$. These price of anarchy can be as large as wanted when $M$ gets large.

As shown in the previous proposition, the quality of Nash equilibria can be very bad if the condition IC is satisfied, or if the profits are strictly positive. We show that if both conditions are fulfilled simultaneously, then the quality of a Nash equilibrium is maximal.

Proposition 4. For strictly positive profits and in the case $I C$, the price of anarchy for problems MaxSP and MaxSP(cov) is 1.

Proof. Assume that all the profits are strictly positive. Then, the only Nash equilibrium is when all the agents co-invest on all the sites. For each site $i, t_{i}^{A}+t_{i}^{B} \geq$ $\max \left(s_{i}^{A}, s_{i}^{B}\right)$, so the co-investment is the strategy with which the total profit is maximum for each site: this corresponds to the optimal solution. The price of anarchy and the price of stability are therefore equal to one.

\begin{tabular}{|c|c|c|}
\hline & Arbitrary profits & Strictly positive profits \\
\hline \multirow{4}{*}{ General case } & $\begin{array}{c}-\nexists \text { Nash equilibrium } \Longleftrightarrow \exists \text { a site } i \text { s.t. } \\
s_{i}^{A}<0<t_{i}^{A} \text { and } t_{i}^{B}<0<s_{i}^{B} \text { (prop 2) }\end{array}$ & - Always a unique Nash equilibrium \\
& - Price of stability unbounded (prop 3) & $\begin{array}{l}\text { Price of stability unbounded } \\
\text { (prop 3) }\end{array}$ \\
\hline Case IC & $-\nexists$ Nash equilibrium $\Longleftrightarrow \exists$ a site $i$ s.t. & - Always a unique Nash equilibrium \\
& $s_{i}^{A}<0<t_{i}^{A}$ and $t_{i}^{B}<0<s_{i}^{B}$ (prop 2) & \\
\hline
\end{tabular}

TABLE 1.Existence and quality of Nash equilibria when the agents do not have

a coverage constraint

Table 1.2 summarizes the results shown in this section. Generally the price of stability is unbounded. To obtain a solution with a better social cost while being accepted by both agents, we introduce a new problem called MaxSP(pmin) which aims to maximize the total amount of profits while providing a minimal benefit to each agent. We show in the following section that in the case $\mathrm{R}$ there exists always a solution in which each agent earns at least as much as it would have received in its best Nash equilibrium. 


\section{When the AGENTS COOPERATE}

We introduce in this section the problems MaxSP(pmin) and MaxSPspec. $\operatorname{MaxSP}(\mathrm{pmin})$ corresponds to the maximization of the overall profits such that each agent earns a minimum value:

$$
\begin{aligned}
\operatorname{maximize} & \sum_{i=1}^{n}\left(x_{i}^{A} \overline{x_{i}^{B}} s_{i}^{A}+\overline{x_{i}^{A}} x_{i}^{B} s_{i}^{B}+x_{i}^{A} x_{i}^{B}\left(t_{i}^{A}+t_{i}^{B}\right)\right) \\
\text { s.t. } & \operatorname{Prof}^{A} \geq P^{A} \\
& \operatorname{Prof}^{B} \geq P^{B}
\end{aligned}
$$

where $\operatorname{Prof}^{A}=\sum_{i=1}^{n}\left(x_{i}^{A} \overline{x_{i}^{B}} s_{i}^{A}+x_{i}^{A} x_{i}^{B} t_{i}^{A}\right)\left(\right.$ resp. $\left.\operatorname{Prof}^{B}=\sum_{i=1}^{n}\left(\overline{x_{i}^{A}} x_{i}^{B} s_{i}^{B}+x_{i}^{A} x_{i}^{B} t_{i}^{B}\right)\right)$ is the profit obtained by Agent $A$ (resp. $B$ ), and where $P^{A}$ (resp. $P^{B}$ ) is the minimum profit that Agent $A$ (resp. $B$ ) must obtain.

Problem MaxSPspec corresponds to the case where $P^{A}$ (resp. $P^{B}$ ) is the maximum profit of agent $A$ (resp. $B$ ) in a Nash equilibrium. Notice that neither $P^{A}$ nor $P^{B}$ are data for an instance of MaxSPspec. However, $P^{A}$ may easily be computed as follows: for any site $i$, the maximal profit of agent $A$ on site $i$ in a Nash equilibrium, $P_{i}^{A}$, is equal to $\max \left\{0, s_{i}^{A}\right\}$ if $t_{i}^{B}<0$, is equal to $\max \left\{0, t_{i}^{A}\right\}$ if $t_{i}^{B}>0$, and is equal to $\max \left\{0, s_{i}^{A}, t_{i}^{A}\right\}$ if $t_{i}^{B}=0$. Hence $P^{A}=\sum_{i=1}^{n} P_{i}^{A}$ can be computed in linear time ( $P^{B}$ is computed in the same way).

We now show in Subsection 2.1 that there is always a solution of the problem MaxSPspec in the case R. Subsection 2.2 studies the complexity of the problem $\operatorname{MaxSP}($ pmin) and shows a dynamic program which solves this problem in pseudopolynomial time.

\subsection{Existence of SOlutions For MaxSPSPEC}

Proposition 5. In the case $R$, there always exists a solution in which Agent $A$ and agent $B$ both earn at least what they would have obtained in any Nash equilibrium.

Proof. Given an instance, we will construct a solution $\mathcal{S}$ in which each agent earns on each site $i$ at least as much as it would have earned on this site in its best Nash equilibrium. Let us consider a site $i$. From Proposition 2, we know that the two following configurations are forbidden when a Nash equilibrium exists: $\left(t_{i}^{A}<0<s_{i}^{A}\right.$ and $\left.s_{i}^{B}<0<t_{i}^{B}\right)$ and $\left(s_{i}^{A}<0<t_{i}^{A}\right.$ and $\left.t_{i}^{B}<0<s_{i}^{B}\right)$.

In the cases where $\left(t_{i}^{A}>0\right.$ and $\left.t_{i}^{B}>0\right)$, in a Nash equilibrium, either the two agents co-invest or no agent invests on the site. When nobody invests, each agent has a profit equal to 0 . When the two agents co-invest, each agent has a positive profit. In the solution $\mathcal{S}$, both agents co-invest for these sites.

Among the other cases, the case where $\left(s_{i}^{A}>0, s_{i}^{B}>0, t_{i}^{A} \leq 0, t_{i}^{B} \leq 0\right)$ is the only one which leads to several Nash equilibria in which the best Nash equilibrium for Agent $A$ is different from the best Nash equilibrium for Agent $B$, and in which the profits perceived by the two agents in their respective best Nash equilibrium 
are not null. In this case, the best Nash equilibrium for Agent $j, j \in\{A, B\}$, is to invest alone on the site. This case is excluded by the definition of case $\mathrm{R}$.

In the cases where $\left(s_{i}^{A} \geq 0, t_{i}^{A} \geq 0, t_{i}^{B}=0\right)$, or $\left(s_{i}^{A} \geq 0, s_{i}^{B}=0, t_{i}^{A} \leq 0, t_{i}^{B} \leq 0\right)$, or $\left(s_{i}^{A}=0, s_{i}^{B}<0, t_{i}^{A} \leq 0, t_{i}^{B} \leq 0\right)$, or $\left(s_{i}^{A}=0, s_{i}^{B} \leq 0, t_{i}^{A}>0, t_{i}^{B}<0\right)$, or $\left(s_{i}^{A}=0, s_{i}^{B}<0, t_{i}^{A}=0, t_{i}^{B}>0\right)$, or $\left(s_{i}^{A}<0, s_{i}^{B}<0, t_{i}^{A} \geq 0, t_{i}^{B} \geq 0\right)$ (and the symmetric cases by exchanging Agents $A$ and $B$ ), there are several Nash equilibria, but the best Nash equilibrium for Agent $A$ and for Agent $B$ correspond (excepted for the case $\left(s_{i}^{A}>0, s_{i}^{B}>0, t_{i}^{A}=0, t_{i}^{B}=0\right)$ excluded just above). In these cases, at least one profit perceived by one of the two agents is null in every Nash equilibria. So, in the solution $\mathcal{S}$, the best Nash equilibrium for Agent $j, j \in\{A, B\}$, is chosen.

In the other cases, there is only one Nash equilibrium. In the solution $\mathcal{S}$, the assignment of the agents on the sites is the same than in the Nash equilibrium.

Therefore, $\mathcal{S}$ is a solution in which each agent earns at least what it would have earn in its best Nash equilibrium.

We have shown that in case $\mathrm{R}$ (which is a realistic case in practice) there is always a solution of the problem $\operatorname{MaxSP}(\mathrm{pmin})$ in which the minimum profit of Agent $A$ (resp. $B$ ) is the maximum profit it may have in a Nash equilibrium. Let us now show that, when the profits are positive, the problem MaxSP(pmin) is NP-hard and can be solved in pseudo-polynomial time.

\subsection{Complexity and algorithm for $\operatorname{MaxSP}(\mathrm{Pmin})$}

Proposition 6. The decision version of the problem MaxSP(pmin) is NP-complete, even if all the profits are non negative.

Proof. We will show that the PARTition problem [6] polynomially reduces to the decision version of $\operatorname{MaxSP}(\operatorname{pmin})$. Let $(E, c)$, where $c: E \rightarrow \mathbb{N}$ and $\sum_{e \in E} c(e)=$ $2 K$, be an instance of PARTITION problem. Let $n=|E|$. The corresponding instance of $\operatorname{MaxSP}(\mathrm{pmin})$ is as follows: with each element $e \in E$ is associated a site $\widehat{e}$. For each site $\widehat{e}, s_{\widehat{e}}^{A}=s_{\widehat{e}}^{B}=c(\widehat{e})$ and $t_{\widehat{e}}^{A}=t_{\widehat{e}}^{B}=0$. Moreover, we assume that Agent $A$ and Agent $B$ must earn at least $K\left(P^{A}=P^{B}=K\right)$.

If $(E, c)$ is a yes instance of the PARTition problem such that $\sum_{e \in E^{\prime}} c(e)=K$, then there is a solution of the corresponding instance of the problem MaxSP(pmin) where Agent $A$ (resp. $B$ ) invests alone on the sites corresponding to the elements of $E^{\prime}$ (resp. $E \backslash E^{\prime}$ ). Each agent has a profit of exactly $K$. Conversely, if there is a feasible solution of the instance of problem $\operatorname{MaxSP}(\mathrm{pmin})$, then in this solution each site is assigned to exactly one agent, and the profit of each agent is exactly $K$ (since the sum of the profits is $2 K)$ : thus, there is a solution to the $(E, c)$ instance of the PARTition problem.

Problem MaxSP(pmin) is NP-hard even if all the profits are non negative. We propose now a dynamic programming algorithm that solves the problem with arbitrary profits. We denote by $P_{A}^{+}=\sum_{i=1}^{n} \max \left\{0, s_{i}^{A}, t_{i}^{A}\right\}\left(\right.$ resp. $\left.P_{A}^{-}=\sum_{i=1}^{n} \min \left\{0, s_{i}^{A}, t_{i}^{A}\right\}\right)$ the upper bound (resp. lower bound) on the total profit of Agent $A$. Similar definitions are given for the bounds $P_{B}^{+}$and $P_{B}^{-}$. 
Given $i \in\{1, \cdots, n\}, g^{A} \in\left\{P_{A}^{-}, \cdots, P_{A}^{+}\right\}$and $g^{B} \in\left\{P_{B}^{-}, \cdots, P_{B}^{+}\right\}$, we denote by $W\left(i, g^{A}, g^{B}\right)$ the global maximal profit that can be obtained for the subproblem associated with the sites $\{1, \cdots, i\}$ when the individual profit of Agent $A$ (resp. agent $B$ ) is exactly $g^{A}$ (resp. $g^{B}$ ). According to these definitions, the value of the

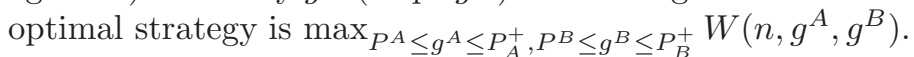

We naturally extend the definition of $W\left(i, g^{A}, g^{B}\right)$ for $i=0$ as follows:

$$
W\left(0, g^{A}, g^{B}\right)= \begin{cases}0 & \text { if } g^{A}=g^{B}=0 \\ -\infty & \text { otherwise }\end{cases}
$$

With these definitions, the recurrence formula followed by $W\left(i, g^{A}, g^{B}\right)$ for $1 \leq$ $i \leq n, g^{A} \in\left\{P_{A}^{-}, \cdots, P_{A}^{+}\right\}$and $g^{B} \in\left\{P_{B}^{-}, \cdots, P_{B}^{+}\right\}$is the following:

$$
W\left(i, g^{A}, g^{B}\right)=\max \left\{\begin{array}{l}
W\left(i-1, g^{A}, g^{B}\right), \\
W\left(i-1, g^{A}-s_{i}^{A}, g^{B}\right)+s_{i}^{A}, \\
W\left(i-1, g^{A}, g^{B}-s_{i}^{B}\right)+s_{i}^{B}, \\
W\left(i-1, g^{A}-t_{i}^{A}, g^{B}-t_{i}^{B}\right)+t_{i}^{A}+t_{i}^{B} .
\end{array}\right.
$$

The optimal strategy is got as usual by recording the optimal decision for each state $\left(i, g^{A}, g^{B}\right)$ and then by collecting these decisions going backward from an optimal final state $\left(n, g_{*}^{A}, g_{*}^{B}\right)$ to the initial state $(0,0,0)$. The complexity of this algorithm is $O\left(n\left(P_{A}^{+}-P_{A}^{-}\right)\left(P_{B}^{+}-P_{B}^{-}\right)\right)$.

\section{EXPERIMENTS}

In this section, we carry out simulations to observe in practice the ratio between the cost in a Nash equilibrium and the cost in an optimal solution, for the problem MaxSP. In the sequel, "price of anarchy" (resp. "price of stability") will denote the average cost, on the instances generated, of the ratio between the cost in the worst (resp. best) Nash equilibrium and the cost in an optimal solution. This is a misnomer because the price of anarchy (resp. price of stability), as explained before, is the maximum ratio, among all the instances, of the worst (resp. best) Nash equilibrium over an optimal solution. Here, we wish to see the average ratio between the cost in the worst (resp. best) Nash equilibrium of an instance and an optimal solution of this instance.

We will also observe the quality of the solutions returned for the problem MaxSPspec. Eventually, we will see to what extent it is possible to improve the profit of each agent by multiplying the profit that each agent earns in its best Nash equilibrium by a constant $\alpha, 1 \leq \alpha \leq 1.5$. We denote this problem by $\operatorname{MaxSPspec}(\alpha)$. These experiments are done on real data provided by Orange Labs, concerning the sites in a developing country, and with randomly generated instances. We first describe the protocol used for randomly generated instances, and then we analyze the results of the simulations. 


\subsection{Protocol}

Profits. We separate the study into two cases: strictly positive profits and arbitrary profits. For each case of profits, we generate several ranges of profits with several types of generators on a number of sites ranging from 1 to 100 by step of 1 . The ranges of profits were $[1 ; 5],[1 ; 100],[1 ; 200]$ and $[300 ; 1000]$ for strictly positive profits, and $[-5 ; 10],[-50 ; 200],[-500 ; 4000]$ and $[-500 ; 100]$ for arbitrary profits. We launched 10000 times the tests, and all the results given are averages.

After studying these different ranges of data with different generators described below, we noticed that the results with strictly positive profits were almost similar. This is the same for arbitrary profits. We have thus retained for further study the range of profits $[1 ; 5]$ for strictly positive profits, and the range of profits $[-5 ; 10]$ for arbitrary profits.

Generators. Four types of profits generators were used:

- Generator 0: there is no restriction on the profits.

- Generator N: this is Generator 0 in which we do not generate the two cases where there is no Nash equilibrium, i.e. there is no site $i$ such that $\left(s_{i}^{A}<0<t_{i}^{A}\right.$ and $\left.t_{i}^{B}<0<s_{i}^{B}\right)$ or $\left(t_{i}^{A}<0<s_{i}^{A}\right.$ and $\left.s_{i}^{B}<0<t_{i}^{B}\right)$.

- Generator IC: this is Generator N in which we add the constraint IC, i.e. for any site $i \in\{1, \ldots, n\},\left(t_{i}^{A}+t_{i}^{B}\right) \geq \max \left(s_{i}^{A}, s_{i}^{B}\right)$.

- Generator R: this is Generator $\mathrm{N}$ in which we add the constraint R, i.e. for any site $i \in\{1, \ldots, n\}$, it is not possible to have $s_{i}^{A}>0, s_{i}^{B}>0, t_{i}^{A} \leq 0$ and $t_{i}^{B} \leq 0$.

As said in the introduction, the generators N, IC and R were created to correspond to real cases. Moreover, the generator $\mathrm{R}$ has also the particularity that it always provides a solution to the problem MaxSPspec, as shown in Proposition 5.

In order to match the real data provided by Orange Labs (cf. the end of this section), the number of persons (subscribers or not) present in the perimeter of each site is generated as a random number between the values 8000 and 30000 .

Computing the solutions. The solution of problem MaxSPspec is computed with the solver IBM ILOG CPLEX. The price of anarchy and the price of stability are easy to compute for the social cost MaxSP, or for MaxSP(cov) with strictly positive profits. Indeed, from one part the optimal solution of problem MaxSP can be computed in $O(n)$, as shown in Section 1.1, and from a another part the best and the worst Nash equilibrium can also be computed in time $O(n)$ (when the profits are strictly positive, the only Nash equilibrium is when both operators always coinvest; when the profits are arbitrary and when there is no cover constraint, it is possible to consider each site independently, and to see for each site which Nash equilibrium is the best or the worst for the sum of the profits). 


\subsection{Results}

Quality of Nash equilibria. For problem MaxSP, we have seen that the price of anarchy and the price of stability are unbounded (see Proposition 3). However, in practice, the price of anarchy is much better since it is less than 1.8, and the price of stability is less than 1.4, and even lower when there are more sites (see Figure 3.2).

For problem MaxSP(cov), when all the profits are strictly positive, the solution where the two agents co-invest on each site is the only Nash equilibrium. As shown in Figures 3.2 and 3.2, the price of stability with the cover constraint is similar to the price of stability without the cover constraint when this latter is smaller than 0.85 (i.e. each agent must cover at most $85 \%$ of the population), and then it decreases when the cover increases (the Nash equilibrium corresponds indeed to the best solution when the cover constraint is 1 ).
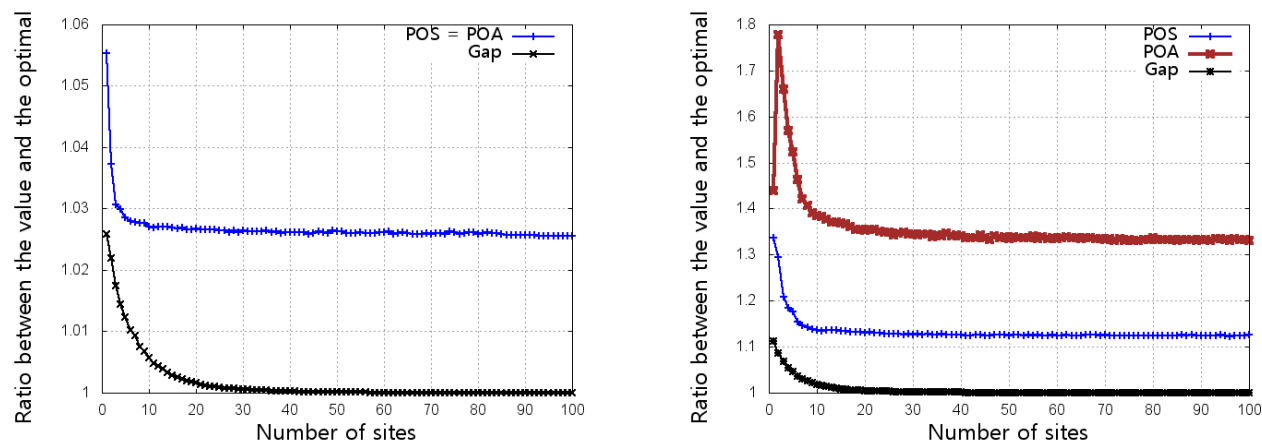

FiguRE 2. For strictly positive profits on the left, and arbitrary profits generated with Generator N on the right: the lower curve ("Gap") represents the ratio between the sum of the profits in an optimal solution of MaxSP and the sum of the profits in an optimal solution of MaxSPspec. The other curves are the price of stability (POS) and the price of anarchy (POA).

Problem MaxSPspec. For strictly positive profits and arbitrary profits generated with Generator R, there is always a solution to the problem MaxSPspec, as seen in Proposition 5. For arbitrary profits generated with the generator N, there are some cases where there is no solution where Agents $A$ and $B$ both obtain what they would obtain in their best Nash equilibrium (see Figure 3.2). This is the case in about $27 \%$ of the instances when there are 7 sites, but this is less often the case with less sites (because instances where there exists a site $i \in\{1, \ldots, n\}$, such that $s_{i}^{A}>0, s_{i}^{B}>0, t_{i}^{A} \leq 0$ and $t_{i}^{B} \leq 0$ are less common), and with more sites (because with many sites it is easier to compensate the loss of one agent on one site with the gain of this agent on another site: for example, the configuration where $s_{i}^{A}>0, t_{i}^{A}>0$ and $s_{i}^{B}>t_{i}^{B}>0$, and where only Agent $B$ is chosen to invest, enables Agent $B$ to compensate a loss of profit on another site).

In the cases where there is a feasible solution of problem MaxSPspec, Figure 3.2 


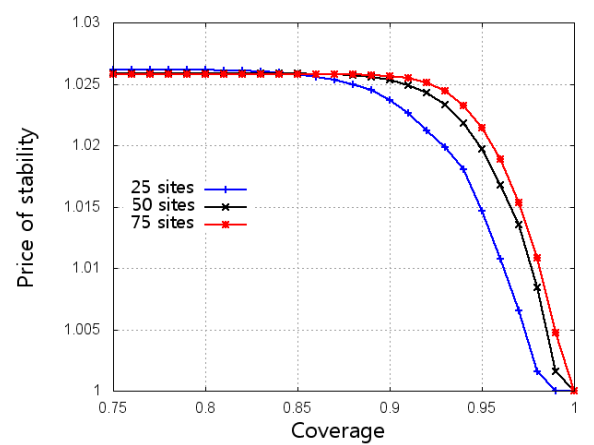

Figure 3. Price of stability for strictly positive profits and with a cover constraint.

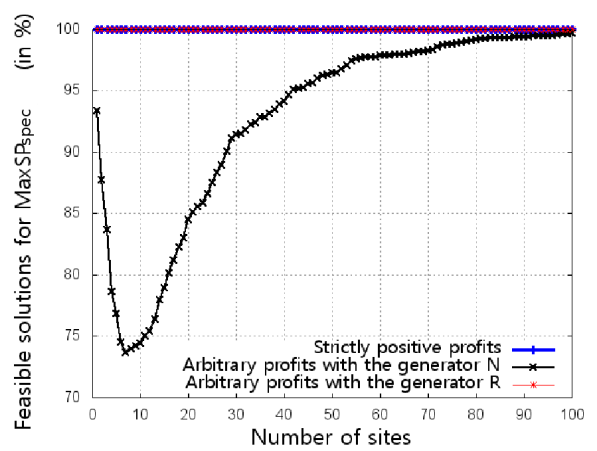

Figure 4. Percentage of times where there is a feasible solution to the problem MaxSPspec.

shows the ratio (noted "Gap") between the value of an optimal solution of problem MaxSP and the value of an optimal solution of problem MaxSPspec. The value of the variable "Gap" is always smaller than the price of stability and is almost equal to 1 when there are more than 10 sites. This highlight the interest of MaxSPspec: even if the solution of this problem is not necessarily a Nash equilibrium, the profit of each agent is larger than or equal to the profit it would have in any Nash equilibrium, and the overall profit is also larger.

Problem MaxSPspec $(\alpha)$. In problem MaxSPspec $(\alpha)$, the aim is to increase the profit of each agent by multiplying the profit that each agent earns in its best Nash equilibrium by a constant $\alpha, 1 \leq \alpha \leq 1.5$. When this value may be larger than or equal to the maximum profit that the agent can have (i.e. for Agent $j, \alpha$ times the profit of Agent $j$ in its best Nash equilibrium is larger than $\left.\sum_{i=1}^{n}\left(\max \left(0, s_{i}^{j}, t_{i}^{j}\right)\right)\right)$, we fix the minimum profit for the agent as $\sum_{i=1}^{n}\left(\max \left(0, s_{i}^{j}, t_{i}^{j}\right)\right)$.

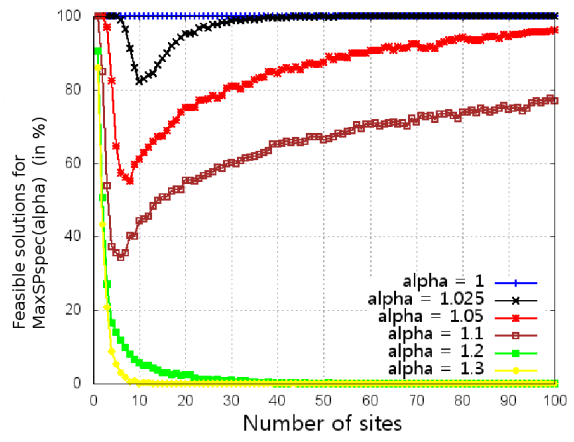

FiguRE 5. For arbitrary profits generated with Generator R, percentage of times where there is a feasible solution to problem $\operatorname{MaxSPspec}(\alpha)$. 
We study the percentage of times where there is a feasible solution to problem $\operatorname{MaxSPspec}(\alpha)$ with different values of $\alpha$ (see Figure 3.2). When $\alpha=1$, there is always a solution, since we use Generator R. For $\alpha=1.025$ to $\alpha=1.1$, the higher the number of sites is, the higher there is a feasible solution (the analysis is the same than in the paragraph concerning problem MaxSPspec). When there is a solution to problem $\operatorname{MaxSP\operatorname {spec}}(\alpha)$, we computed the ratio between the value of the optimal solution of MaxSP and the value of the optimal solution of $\operatorname{MaxSPspec}(\alpha)$. The results are similar to what we obtained without multiplying the profit of each agent by $\alpha$ (these results were shown on Figure 3.2).

Real data. Real data from a developing country, provided by Orange Labs, contain 28 sites and, for each site, the population covered and the number of Orange's subscribers. We fix a cost of investment, a cost of co-investment and the price for phone subscription. These numbers are the same for each site and each operator (agent). We simulate the number of clients of the second agent. We consider four cases: this second agent has the same number of clients as Orange, $30 \%$ less, $30 \%$ more, or the rest of the possible clients on each site (i.e., the population covered minus the number of Orange's subscribers). The profit of Agent $j$ on site $i$ corresponds to the number of clients that subscribe to Agent $j$ multiplied by the price of phone subscription, minus the cost of investment of site $i$. Table 1 shows the price of anarchy, the price of stability, and the ratio ("Gap") between the value of the optimal solution of MaxSP and the value of the optimal solution of MaxSPspec. As shown in this table, in practice, the price of anarchy and the price of stability are very low, but are still larger than or equal to the optimal solution of MaxSPspec.

\begin{tabular}{|c|c|c|c|}
\hline Number of clients of the second agent & Price of stability & Price of anarchy & Gap \\
\hline The remaining clients & 1.021 & 1.023 & 1.02 \\
\hline Same number as Orange & 1 & 1.163 & 1 \\
\hline $30 \%$ less than Orange & 1.152 & 1.152 & 1.152 \\
\hline $30 \%$ more than Orange & 1.045 & 1.069 & 1.044 \\
\hline
\end{tabular}

TABLE 1. Results on instances obtained from real data.

\section{Conclusion}

In this work, we have considered the problem of sites sharing by two selfish telecommunication operators. We showed that there always exists a (pure) Nash equilibrium, except if the profits follow a very unrealistic configuration. We introduced problem MaxSP(pmin), which is NP-hard but which can be solved in pseudo-polynomial time with a dynamic program. We showed that for realistic configurations (case R) it is always possible to return a solution (the optimal solution of MaxSPspec) in which each agent earns at least what it would earn in a 
Nash equilibrium. We believe that the agents should agree to accept such a solution, proposed by a central entity. We conducted experiments which show that the price of anarchy and the price of stability are in practice much better than in the worst case (where it is generally unbounded), and that the ratio between the social cost and the value of an optimal solution of $\operatorname{MaxSP}(\mathrm{pmin})$ is smaller than the price of stability.

Several future work are possible: in the experimentations, we focus on situations where Nash equilibria can be found easily, which represent all the cases except the case where there is a cover constraint and when some of the profits are negative. In this case it is NP-hard to find a Nash equilibrium, if such a Nash equilibrium exists, since it is even NP-hard for an agent alone to choose where to invest. It would nevertheless be interesting to study the conditions of existence of Nash equilibria in this case, and to see whether introducing a problem such as MaxSPspec with cover constraints could be useful. It would also be interesting to extend the model to a constant number of agents. More generally, we believe that an algorithm returning a solution of good quality for a social cost and in which each agent has a profit larger than or equal to what it would have had in any Nash equilibrium can be useful in other problems in which the utility is not transferable.

Eventually, this work is a first step towards the study of the advantages of cooperation for site sharing between telecommunication operators. Additional constraints could be added to get closer to real situations.

\section{REFERENCES}

[1] E. Anshelevich, A. Dasgupta, J. Kleinberg, É. Tardos, T. Wexler, and T. Roughgarden. The price of stability for network design with fair cost allocation. In Symposium on Foundations of Computer Science (FOCS), pages 295-304, 2004.

[2] A. Barlett and N. N. Jackson. Network planning considerations for network sharing in umts. In Third International Conference on 3G Mobile Communication Technologies, pages 1721. Motorola UK, 2002.

[3] J. Cohen, D. Cordeiro, D. Trystram, and F. Wagner. Multi-organization scheduling approximation algorithms. In Concurrency and Computation: Practice and Experience, volume 23(17), pages 2220-2234, 2011.

[4] D. Cordeiro, P. F. Dutot, G. Mounié, and D. Trystram. Tight analysis of relaxed multiorganization scheduling algorithms. In 25th IEEE International Parallel and Distributed Processing Symposium (IPDPS), pages 1177-1186, 2011.

[5] P. F. Dutot, F. Pascual, K. Rzadca, and D. Trystram. Approximation algorithms for the multi-organization scheduling problem. In IEEE Transactions on Parallel and Distributed Systems, volume 22(11), pages 1888-1895, 2011.

[6] M. R. Garey and D. S. Johnson. A Guide to the Theory of NP-Completeness.

[7] L. Gourvès, J. Monnot, and F. Pascual. Cooperation in multiorganization matching. In 6th International Workshop of Approximation and Online Algorithms, volume 5426, pages 78-91, 2009.

[8] E. Koutsoupias and C. Papadimitriou. Worst-case equilibria. In Symposium on Theoretical Aspects of Computer Science (STACS), volume 1563 of LNCS, pages 404-413. Springer, 1999.

[9] J. Nash. Non-cooperative games. In Annals of Mathematics, volume 54 of Second Series, pages 286-295, 1951. 
[10] T. Norman and C. Viola. Transform the economics of your wireless business with infrastructure sharing. Technical report, Analysys Mason, April 2010.

[11] F. Ooshita and T. Izumi. A generalized multi-organization scheduling on unrelated parallel machines. In International Conference on Parallel and Distributed Computing, Applications and Technologies, pages 26-33, 2009.

[12] M. J. Osborne and A. Rubinstein. A Course in Game Theory. The MIT Press, Cambridge, Massachusetts, London, England, 1994.

[13] F. Pascual, K. Rzadca, and D. Trystram. Cooperation in multi-organization scheduling. In Euro-Par, Parallel Processing, volume 4641, pages 224-233, 2007.

[14] W. Saad, Z. Han, M. Debbah, A. Hjorungnes, and T. Basar. Coalitional game theory for communication networks. Signal Processing Magazine, 5:77-97, 2009.

[15] A. S. Schulz and N. S. Moses. On the performance of user equilibria in traffic networks. In Symposium on Discrete Algorithms (SODA), pages 86-87, 2003. 